TITLE:

\title{
Facilitated Transport of lons and Glucose by Amphotericin B Across Lipid Bilayers in the Presence or Absence of Cholesterol
}

\section{$\operatorname{AUTHOR}(S):$}

Kagohashi, Hiroki; Shirai, Osamu; Kubota, Shintaro; Kitazumi, Yuki; Kano, Kenji

\section{CITATION:}

Kagohashi, Hiroki ...[et al]. Facilitated Transport of Ions and Glucose by Amphotericin B Across Lipid Bilayers in the Presence or Absence of Cholesterol. Electroanalysis 2014, 26(3): 625-631

\section{ISSUE DATE:}

2014-03

URL:

http://hdl.handle.net/2433/199597

\section{RIGHT:}

This is the peer reviewed version of the following article: Kagohashi, H., Shirai, O., Kubota, S., Kitazumi, Y. and Kano, K. (2014), Facilitated Transport of Ions and Glucose by Amphotericin B Across Lipid Bilayers in the Presence or Absence of Cholesterol. Electroanalysis, 26: 625-631, which has been published in final form at

http://dx.doi.org/10.1002/elan.201300555. This article may be used for non-commercial purposes in accordance with. Wiley Terms and Conditions for Self-Archiving.: この論文は出版社版でありません。引用の際には出版社版をご確認ざ 利用ください。; This is not the published version. Please cite only the published version. 
Facilitated Transport of Ions and Glucose by Amphotericin B across Lipid Bilayers in the Presence or Absence of Cholesterol

Hiroki Kagohashi, Osamu Shirai*, Shintaro Kubota, Yuki Kitazumi, and Kenji Kano

Division of Applied Life Sciences, Graduate School of Agriculture, Kyoto University, Sakyo, Kyoto 606-8502, Japan.

Keywords: Amphotericin B; Bilayer lipid membrane; Ion transport; Liposome; Glucose; Cholesterol

*Corresponding Author. Address: Division of Applied Life Sciences, Graduate School of Agriculture, Kyoto University, Sakyo, Kyoto 606-8502, Japan. Tel: +81-75-753-6394; Fax: $+81-75-753-6456$

E-mail address: shiraio@kais.kyoto-u.ac.jp (O. Shirai). 


\begin{abstract}
The transport of ions and glucose across bilayer lipid membranes (BLM) facilitated by amphotericin B (AmB) is studied by use of planar BLMs and liposomal membranes. The transport characteristics change with time in the presence of cholesterol, while it is independent of time in the absence of cholesterol. The carrier-type transport is observed immediately after the addition of AmB. In the presence of cholesterol, AmB forms a 1:1 complex with cholesterol and the channel is formed by aggregation of AmB-cholesterol complexes. It is concluded that the number of the channels increases with time and that the carrier-type transport decreases instead.
\end{abstract}




\section{Introduction}

Amphotericin B (AmB) is a polyene antibiotic derived from Streptomyces nodosus and it is widely utilized for treatment of systemic fungal infections [1,2]. It is well known that AmB associates with ergosterol or cholesterol in the ratio of one to one in biomembranes and that plural AmB-sterol complexes aggregate to form transmembrane channels [3-6]. It is generally accepted that fungi become extinct by the leakage of substances from the inside of the fungic cells on the addition of AmB, since most fungi usually have ergosterol [1,2]. Because the human body scarcely has ergosterol, it is expected that the side effects of AmB are caused by the presence of cholesterol. The permeability of not only neutral compounds such as water, glucose, and urea but also monovalent ions such as $\mathrm{K}^{+}, \mathrm{Na}^{+}$, and $\mathrm{Cl}^{-}$across $\mathrm{AmB}$ channels has been reported by many groups [7-10]. In addition, the formation of several types of AmB channels has been described $[3,7,8,11,12]$. Understanding of detailed properties of AmB is very important for treatment of systemic fungal infections. We consider that for this purpose it is essential to characterize the transport of both ions and neutral compounds through the AmB-containing membrane.

In our group, an analytical method on channel properties by use of the planar BLM system has been reported [13,14]. It is a powerful method to evaluate the amounts of transported ions and their permselectivity. As for a channel-forming peptide gramicidin A (GA), it has been found that GA serves as not only a channel-type transporter but also a carrier-type transporter. By varying the concentration of the objective electrolyte in aqueous phases, the physicochemical properties of the ion transport have been evaluated in detail. On the other hand, it is impossible to evaluate the amount of neutral compounds transferred across the BLM in a similar manner as above, since they do not have electric charges. In the case of transport of glucose which is one of neutral compounds, the observation of the leakage of glucose from the 
inside of liposomes seems to be effective in the evaluation of the transport property, since it is easy to detect glucose in the outside solution by amperometric sensors [15].

In the present work, the influence of $\mathrm{AmB}$ on the transport of potassium chloride as an electrolyte and glucose as a non-electrolyte across bilayer lipid membranes in the presence and absence of cholesterol is investigated by considering the electroneutrality and the mass balance in the whole phases.

\section{Experimental}

\subsection{Chemicals}

The lipids used for BLMs and liposomal membranes were lecithin (Wako Pure Chem., Ind., Ltd.) and cholesterol (Sigma-Aldrich Co., Ltd.). AmB was purchased from Wako Pure Chem. Ind., Ltd. All other reagents were of reagent grade and used without further purification.

\subsection{Measurements of voltammograms for the ion transport across BLMs and thin liquid} membranes

The electrochemical cell used for the BLM system was the same as that used in previous works [13,14]. Two aqueous phases (W1 and W2) were filled with $15 \mathrm{~mL}$ of aqueous solutions and were separated with a 0.2-mm thick tetrafluoroethylene resin sheet (Mitsui Fluorochemical Co.). BLMs were obtained by brushing an $n$-decane solution of the lipids on a $1.0-$ mm diameter aperture created on the tetrafluoroethylene resin sheet. The formation of BLMs was confirmed by capacitance measurements and microscopic observations of the internal reflection from the membrane surface. The BLM-forming solution was prepared by dissolving $10 \mathrm{mg}$ of lecithin and $2.5 \mathrm{mg}$ of cholesterol into $n$-decane in a $1-\mathrm{mL}$ flask. The aqueous solutions contained 10 $\mathrm{mM}$ 4-(2-hydroxyethyl)-1-piperazineethanesulfonic acid (HEPES) and $10 \mathrm{mM}$ 
bis(2-hydroxyethyl)iminotris-(hydroxymethyl)methane (Bis-Tris) mixtures (pH 7.4). AmB was dissolved into dimethyl sulfoxide (DMSO) in advance to prepare $1.0 \mathrm{mM}$ AmB stock solution. The AmB solution was added into both two aqueous phases (W1 and W2) to reach the objective concentration.

The electrochemical cell used for the thin liquid-membrane (tLM) system was the same as that for the BLM system. Instead of the tetrafluoroethylene resin sheet, a porous tetrafluoroethylene resin sheet impregnating $n$-decane containing $4 \mathrm{mg} \mathrm{mL}^{-1}$ lecithin and $2 \mathrm{mg}$ $\mathrm{mL}^{-1}$ cholesterol was used as a separator between $\mathrm{W} 1$ and $\mathrm{W} 2$. The surface area of the tLM was $1 \mathrm{~cm}^{2}$ and the thickness was $75 \mu \mathrm{m}$.

The voltammogram for the ion transport between W1 and W2 across the BLM or the tLM was recorded by scanning the potential difference between $\mathrm{W} 1$ and $\mathrm{W} 2\left(E_{\mathrm{W} 1-\mathrm{W} 2}\right)$ and by measuring the current between W1 and W2 (I $\left.I_{\mathrm{W} 1-\mathrm{W} 2}\right)$. $E_{\mathrm{W} 1-\mathrm{W} 2}$ was applied through two Ag|AgCl electrodes (in $0.1 \mathrm{M} \mathrm{KCl}$; RE1 and RE2) in $\mathrm{W} 1$ and $\mathrm{W} 2$, while $I_{\mathrm{W} 1-\mathrm{W} 2}$ was measured with two platinum wire electrodes (CE1 and CE2) in W1 and W2. In this work, the positive current was defined as the current due to both the transfer of positively charged species from W1 to W2 and that of negatively charged species from W2 to W1. The ion transport current density, $j_{\mathrm{w} 1-\mathrm{w} 2}$, was obtained by dividing $I_{\mathrm{W} 1-\mathrm{w} 2}$ by the area of the BLM or the tLM. The voltammograms were recorded using a potentiostat Model HA1010mM1A (Hokuto Denko Co.) and a function generator Model HB-305 (Hokuto Denko Co.). The electrochemical cell was placed in a Faraday cage during the measurements in order to decrease background electric noises. All measurements were performed at room temperature (298 $\pm 3 \mathrm{~K})$.

\subsection{Preparation of liposome solutions}

The mixture of $40 \mathrm{mg}$ of lecithin and $10 \mathrm{mg}$ of cholesterol in a round-bottom flask was 
dissolved into approximately $30 \mathrm{~mL}$ chloroform, and chloroform was removed by a rotary evaporator Model N-1000 (Eyela Co.) to obtain a thin film on the inside surface of the flask. The lipid film was suspended in $5 \mathrm{~mL}$ of $10 \mathrm{mM}$ sodium phosphate buffer solution (NaPB, $\mathrm{pH}$ 7.4) containing $30 \mathrm{mM}$ glucose or $50 \mathrm{mM} \beta$-nicotinamide adenine dinucleotide disodium salt $\left(\mathrm{NAD}^{+}\right)$. Then the lipid suspension was frozen in liquid nitrogen and was thawed to enhance the formation of large multi-lamellar vesicles (MLVs). This freezing and thawing cycle was carried out three times. MLVs were transformed to unilamellar liposomes by an extruder Avanti Mini-Extruder (Avanti Polar Lipid, Inc.), and the unilamellar liposomes with a mean outside diameter of approximately $100 \mathrm{~nm}$ were prepared. Since glucose or $\mathrm{NAD}^{+}$remained in the outside solution of the liposomes, the liposomes were separated from the outside solution by passing through a column of Sephadex G-50 (GE Healthcare).

\subsection{Observation of the outflow of glucose or NADH from liposomes}

The glucose concentration in the outside solutions of the liposomes was monitored by amperometry with a glucose oxidase/peroxidase/ferrocene-immobilized electrode. This electrode was fabricated according to a previous work [15], and the details are given in SI. As for the detection of NADH in the outside solutions of the liposomes, a glucose dehydrogenase/1-methoxy PMS-immobilized electrode of which the fabrication method is noted in SI was used.

The enzyme-immobilized electrodes, an $\mathrm{Ag} \mid \mathrm{AgCl}$ electrode (in saturated $\mathrm{KCl}$ ) and a $\mathrm{Pt}$ wire were used for the working, reference and counter electrodes, respectively. Amperometric studies were performed on an ALS CHI611A electrochemical analyzer. In case of $\mathrm{NAD}^{+}$ measurements, NaPB was used as an electrolyte solution containing $50 \mathrm{mM}$ glucose (pH 7.4). The applied potentials for the measurement of glucose and $\mathrm{NAD}^{+}$were 0 and $300 \mathrm{mV}$ vs. the 
$\mathrm{Ag} \mid \mathrm{AgCl}$ electrode, respectively. After AmB was added into the liposome suspensions (the final concentration $=10 \mu \mathrm{M})$, the concentration of glucose or $\mathrm{NAD}^{+}$was monitored chronoamperometrically. In this work, AmB was dissolved into dimethyl sulfoxide (DMSO) in advance, and it was used as a stock solution. At the end of the measurements, Triton X-100 was added into the liposome suspension at final concentrations of $0.1-0.2$ wt.\% to completely break the liposomes.

The above measurements were carried out under moderate stirring with a magnetic stirrer at $278 \pm 1 \mathrm{~K}$.

\section{Results and Discussion}

\subsection{Ion transport across BLMs in the absence or presence of cholesterol}

Figure 1 shows cyclic voltammograms for the ion transport across the cholesterol-free BLMs in the presence of $\mathrm{AmB}$ between $\mathrm{W} 1$ containing $0.02 \mathrm{M} \mathrm{KCl}$ and $\mathrm{W} 2$ containing $0.01 \mathrm{M} \mathrm{KCl}$. Curves 1, 2 and 3 were started at 0,120 and $200 \mathrm{~s}$ after the addition of AmB. The membrane potential at $j_{\mathrm{w} 1-\mathrm{w} 2}=0$, which is named the zero-current potential $\left(E_{\mathrm{W} 1-\mathrm{W} 2, j=0}\right)$, was about $-23 \mathrm{mV}$, as shown by the inset of Fig. 1 . The magnitude of $j_{\mathrm{w} 1-\mathrm{w} 2}$ at a given $E_{\mathrm{W} 1-\mathrm{W} 2}$ increased with time by $1200 \mathrm{~s}$ later. Because the concentration of $\mathrm{KCl}$ in $\mathrm{W} 1$ was higher than that in $\mathrm{W} 2$, both $\mathrm{K}^{+}$

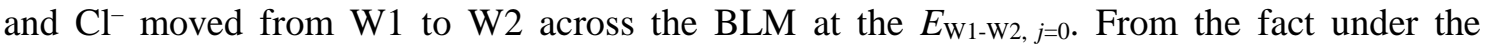
situation that the $E_{\mathrm{W} 1-\mathrm{W} 2, j=0}$ value is negative, and we can conclude that the transport of $\mathrm{K}^{+}$ across the BLM is much faster than that of $\mathrm{Cl}^{-}$in the case of the cholesterol-free BLM [16]. At any $E_{\mathrm{W} 1-w 2}$, the current fluctuation due to the channel-type transport was not observed.

Normal pulse voltammograms (lines 1-3), which were obtained to decrease the influence of charging current, for the ion transport across the tLM is depicted in Fig. 2. The current density in the presence of $\mathrm{AmB}$ (line 2) was larger than that in the absence of AmB (line 1). 
Since the thickness of the tLM $(75 \mu \mathrm{m})$ is much larger than that of the BLM (about $6 \mathrm{~nm}$ ), AmB cannot form any channels penetrating the tLM. Therefore, it is clear that the facilitation of the ion transport current is caused by the AmB-induced carrier-type transport. Because the carrier-type transport is observed even when a channel-forming compound (gramicidin A) is used as a transporter $[13,14]$, it can be concluded that the ion transport across the BLM is ascribed to the carrier-type transport. When the concentration of $\mathrm{KCl}$ in $\mathrm{W} 1$ was 10 times higher than that in W2 (line 3), the $E_{\mathrm{W} 1-\mathrm{W} 2, j=0}$ value was about $-40 \mathrm{mV}$. Since the junction potential between W1 and RE1 was negligibly small, the negative shift of the $E_{\mathrm{W} 1-\mathrm{W} 2, j=0}$ is attributable to the difference between the transport number of $\mathrm{K}^{+}$and that of $\mathrm{Cl}^{-}$. This means that the carrier-type transport in the tLM system has the cation selectivity.

On the other hand, it has been widely accepted that AmB forms channels in the presence of cholesterol $[3,9,10,17]$. Curves 1,2 and 3 of Fig. 3 are cyclic voltammograms for the ion transport across the cholesterol-containing BLMs between W1 containing $0.03 \mathrm{M} \mathrm{KCl}$ and 500 $\mathrm{nM}$ AmB and W2 containing $0.01 \mathrm{M} \mathrm{KCl}$ and $500 \mathrm{nM} \mathrm{AmB}$. The start times of curves 1, 2 and 3 were 0,30 and $120 \mathrm{~s}$, respectively, after the addition of $\mathrm{AmB}$. The $E_{\mathrm{W} 1-\mathrm{w} 2, j=0}$ at the first scan was about $-10 \mathrm{mV}$, and the $E_{\mathrm{W} 1-\mathrm{W} 2, j=0}$ value shifted in the positive direction with time. The $E_{\mathrm{W} 1-\mathrm{W} 2, j=0}$ at the third scan showed about $+20 \mathrm{mV}$. Simultaneously, $j_{\mathrm{W} 1-\mathrm{W} 2}$ at a given $E_{\mathrm{W} 1-\mathrm{W} 2}$ decreased with time up to $120 \mathrm{~s}$. After $120 \mathrm{~s}$ from the addition of $\mathrm{AmB}$, the $E_{\mathrm{W} 1-\mathrm{W} 2, j=0}$ value was kept constant at about $20 \mathrm{mV}$ but the current continuously increased with time, as shown by curves 3, 4 and 5 in Fig. 3. The current due to the channel transport maintained for more than several hours at least. This result indicates that the main transport mechanism was changed from the carrier-type transport to the channel-type transport around $120 \mathrm{~s}$ after the addition of $\mathrm{AmB}$ and that the ion transport property changed from the cation permselectivity to the anion permselectivity. Since AmB molecules spontaneously distribute from $\mathrm{W} 1$ and $\mathrm{W} 2$ to the BLM, AmB might initially 
serve as a carrier. The number of $\mathrm{AmB}$ channels seems to increase with time by the aggregation

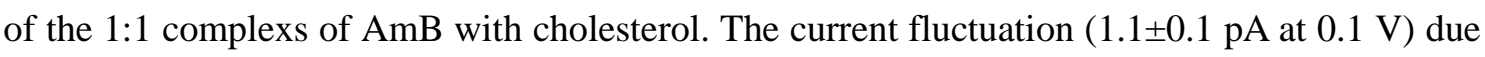
to the channel transport was observed when $\mathrm{W} 1$ and $\mathrm{W} 2$ contained $0.3 \mathrm{M} \mathrm{KCl}$ and $10^{-9} \mathrm{M} \mathrm{AmB}$ (Fig. S1). Therefore, it is considered that the time-dependent characteristics are attributed to the change in the transport mechanism from the carrier type to the channel type.

\subsection{Glucose transport across liposomal membranes}

\subsubsection{The time dependence of glucose transport by $\mathrm{AmB}$ in the presence of cholesterol}

At first, in order to make sure that the liposomal membranes remain unbroken on the addition of $\mathrm{AmB}, \mathrm{NAD}^{+}$, of which the size is much larger than channel pore ( $0.8 \mathrm{~nm}$ in the inside diameter), was used as a marker solute inserted into the liposomes [19]. The detection of $\mathrm{NAD}^{+}$which flowed out of the liposomes was performed by amperometry with a glucose dehydrogenase/1-methoxy PMS immobilized electrode. Regardless the presence or the absence of cholesterol, the outflow of $\mathrm{NAD}^{+}$from the inside of the liposomes to the outside was not

Fig. S2 completely detected throughout the measurements (Fig. S1). Since $\mathrm{NAD}^{+}$could not be transferred from the inside to the outside, it is concluded that liposomes are not broken on the addition of AmB.

When $1.0 \mathrm{mM} \mathrm{AmB}$ in DMSO was added to the liposome suspension until the final concentration became $10 \mu \mathrm{M}$, glucose encapsulated within the liposomes started to flow out of the liposomes. Therefore, the glucose concentration in the outside solution of the liposomes increased with time and reached a constant value within $200 \mathrm{~s}$ in the presence of cholesterol in the liposomal membranes, as presented by curve 1 of Fig. 4 . After the glucose concentration became constant, Triton $\mathrm{X}-100$ was added to break the liposomal membranes. As a result, the concentration of glucose immediately increased up to about $150 \mu \mathrm{M}$. Here, the final glucose 
concentration after the addition of Triton $\mathrm{X}-100$ is defined as $c^{\prime} \infty$. The broken line indicates a hypothetical curve based on a simple diffusion model in consideration of an internal and external concentration difference and an internal and external volume ratio $\left(c=c^{\prime} \infty\right.$ [1-exp(-k't)], $k^{\prime}$ : the rate constant) [18], where the $k^{\prime}$ value was estimated from the initial parts of the outflow curve. These results demonstrate that the glucose transport by AmB is depressed within about $200 \mathrm{~s}$ after the addition of AmB.

If glucose would not move through the AmB channel, we predict that the outflow of glucose stops about several hundred seconds after the addition of AmB. In fact, the outflow of glucose stopped by about $200 \mathrm{~s}$ after the addition of AmB, as indicated in Fig. 4 (A). It is clear that the ion transport was controlled by the AmB channel, and the channel activity held for more than several hours. Although about $50 \%$ of glucose enclosed in the liposomes was leaked by $200 \mathrm{~s}$, the glucose concentration in the outside was almost constant since then. The liposome suspension containing $10 \mu \mathrm{M}$ of $\mathrm{AmB}$ was left at $277 \mathrm{~K}$ for $13 \mathrm{~h}$, and then Triton $\mathrm{X}-100$ was added into the liposome suspension to completely break the liposomes. The time course of the glucose concentration in the outside solution of the liposomes in the presence of cholesterol after the addition of Triton $\mathrm{X}-100$ is depicted in the inset of Fig. 4 (A). The glucose concentration increased after the addition of Triton X-100. Consequently, it is concluded that glucose molecules cannot pass through the AmB channels.

\subsubsection{The time dependence of glucose transport by AmB in the absence of cholesterol}

As for the cholesterol-free system, the glucose concentration in the outside solution of the liposomes slowly increased as shown in Fig. 4 (B). When Triton X-100 was added $13 \mathrm{~h}$ after the addition of AmB, the glucose concentration scarcely increased. This indicates that glucose is in equilibrium between the inside and outside of the liposomes. However, the increase in the 
rate of the glucose transport in the outside solution of the liposomes was much smaller than the predicted values (the broken line in Fig. 4 (B)), which were evaluated based on the simple diffusion model. This unexpected drop in the outflow rate might be caused by the aggregation of AmB in the aqueous phase, as described below.

The inset (A) of Fig. 5 shows absorption spectrum of the DMSO solution of $10 \mu \mathrm{M}$ AmB. There are three peaks at 369, 390 and $414 \mathrm{~nm}$, which are ascribed to the monomeric form of AmB [20,21]. On the other hand, the inset (B) of Fig. 5 indicates absorption spectrum of $10 \mu \mathrm{M}$ AmB dissolved in the NaPB solution (pH7.4) containing 1.0 \% DMSO. In the NaPB solution, another peak at $326 \mathrm{~nm}$ derived from the aggregated form of AmB is observed [20,21]. Curves 1 and 2 of Fig. 5 represent time dependence of the absorbance peaks at $408 \mathrm{~nm}$ and $326 \mathrm{~nm}$ in the NaPB solution (pH7.4) after the addition of AmB at an initial concentration of $10 \mu \mathrm{M}$. The absorbance at $408 \mathrm{~nm}$ decreased with time while the absorbance at $326 \mathrm{~nm}$ increased during the initial period of $200 \mathrm{~s}$. Since the aggregation of AmB molecules proceeds, the monomeric form of AmB decreased on the same time scale with the decrease in the glucose transport rate in the cholesterol-free system. Therefore, we considered two mechanisms which can explain the decrease in the glucose transport rate in the cholesterol-free system: 1) the rate of distribution of AmB to the liposomal membranes from the aqueous solution is larger than the rate of aggregation of $\mathrm{AmB}$ in the aqueous solution; and 2) only monomeric form of $\mathrm{AmB}$ can distribute to the liposomal membranes. The amount of AmB molecules distributed to the membranes decreases with time after the addition of AmB dissolved in DMSO because the amount of the monomeric form of $\mathrm{AmB}$ in the aqueous solution decreases as its aggregation proceeds with time. Then, the NaPB solution (pH7.4) containing $1.0 \mathrm{mM} \mathrm{AmB}$ and $10 \%$ DMSO was prepared in order to form AmB-aggregates in advance, and it was added to the liposome suspension instead of the direct addition of $1.0 \mathrm{mM} \mathrm{AmB}$ in DMSO (as the 
monomeric form) to the cell. The rate of the glucose-efflux was smaller than that in the case of direct addition of the DMSO solution containing AmB as shown in Fig. S3, and the unexpected decrease of the outflow rate was not observed in this case. These results show that the unexpected decrease in the rate of the glucose-efflux is mainly caused by the aggregation of AmB in the external aqueous phase of the liposome suspension.

\section{Conclusion}

It has been clarified that AmB works not only as a carrier-type transporter but also as a channel-type transporter. The AmB-induced transport mechanism of ions and glucose across the BLMs changed with time in the cholesterol-containing system, while that in the cholesterol-free system remain unchange. AmB works as carriers at first, and then they aggregate with time to form channels in the presence of cholesterol. On the other hand, AmB works only as carriers in the absence of cholesterol. However, in the absence of cholesterol, the rate of the outflow of glucose from the liposomes decreased with time. It is concluded that the aggregation of AmB molecules in the outside aqueous phase decreases the concentration of the monomeric form to reduce the glucose transport rate across the liposomal membranes. 


\section{References}

[1] J. Brajtburg, W. G. Powderly, G. S. Kobayashi, G. Medoff, Antimicrob. Agents Chemother., 1990, 34, 183.

[2] H. A. Gallis, R. H. Drew, W. W. Pickard, Rev. Infect. Dis., 1990, 12, 308.

[3] B. De Kruijff, R.A. Demel, Biochim. Biophys. Acta, 1974, 339, 57.

[4] A. Marty, A. Finkelstein, J. Gen. Physiol., 1975, 65, 515.

[5] N. Matsumori, K. Tahara, H. Yamamoto, A. Morooka, M. Doi, T. Oishi M. Murata, J. Am. Chem. Soc., 2009, 131, 11855.

[6] A. Neumann, M. Baginski, J. Czub, J. Am. Chem.l Soc., 2010, 132, 18266.

[7] J. Bolard, Biochim. Biophys. Acta, 1986, 864, 257.

[8] B. E. Cohen, Biochim. Biophys. Acta, 1986, 857, 117.

[9] V. Khutorsky, Biophys. J., 1996, 71, 2984.

[10] G. Fujii, J.-E. Chang, T. Coley, B. Steere, Biochemistry, 1997, 36, 4959.

[11] A. Cass, A. Finkelstein, V. Krespi, J. Gen. Physiol., 1970, 56, 100.

[12] B. V. Cotero, S. Robolledo-Antúnez, I. Ortego-Blake, Biochim. Biophys. Acta, 1998, 1375, 43.

[13] O. Shirai, Y. Yoshida, S. Kihara, T. Ohnuki, A. Uehara, H. Yamana, J. Electroanal. Chem., 2006, 595, 53.

[14] S. Kubota, S. Ozaki, J. Onishi, K. Kano, O. Shirai, Anal. Sci., 2009, 25, 189.

[15] H. Kinoshita, M. Torimura, K. Kano, T. Ikeda, Electroanalysis, 1997, 9, 1234.

[16] J. Onishi, O. Shirai, K. Kano, Electroanalysis, 2010, 22, 1229.

[17] T. E. Andreoli, Ann. N. Y. Acad. Sci., 1974, 235, 448.

[18] R. Holtz, A. Finkelsein, J. Gen. Physiol., 1970, 56, 125.

[19] A. J. Bard, L. R. Faulkner, Electroanalytical Chemistry, 2nd ed., John Wiley \& Sons, Inc., 
New York, 1967.

[20] J. Bolard, M. Seigneuret, G. Boudet, Biochim. Biophys. Acta, 1980, 599, 280.

[21] C. Ernst, J. Grange, H. Rinnert, G. Dupont, and J. Lematre, Biopolymer, 1981, 20, 1575. 


\section{Figure captions:}

Fig. 1

Cyclic voltamograms for the ion transport across a cholesterol-free BLM in the presence of 50 $\mu \mathrm{M}$ AmB. Start times of the potential sweep: 0, 120 and $200 \mathrm{~s}$ (curves 1, 2, and 3) after the addition of AmB. Potential scanning rate: $10 \mathrm{mV} \mathrm{s}^{-1}$. Electrolyte in $\mathrm{W} 1: 0.02 \mathrm{M} \mathrm{KCl}, 0.01 \mathrm{M}$ HEPES and 0.01 M Bis-Tris. Electrolyte in W2: 0.01 M KCl, 0.01 M HEPES and 0.01 M Bis-Tris. $\mathrm{pH}=7.4 . T=298 \pm 3 \mathrm{~K}$. The inset shows the time dependence of $j_{\mathrm{w} 1-\mathrm{w} 2}(1)$ and $E_{\mathrm{W} 1-\mathrm{W} 2, j=0}(2)$.

Fig. 2

Normal pulse voltamograms for the ion transport across tLMs in the absence of AmB (line 1) and the presence of $50 \mu \mathrm{M} \mathrm{AmB}$ (lines 2 and 3). Electrolyte in W1: $0.01 \mathrm{M} \mathrm{KCl}$ (lines 1 and 2) or $0.1 \mathrm{M} \mathrm{KCl}$ (line 3), $0.01 \mathrm{M}$ HEPES and $0.01 \mathrm{M}$ Bis-Tris. Electrolyte in W2: $0.01 \mathrm{M} \mathrm{KCl}$, 0.01 M HEPES and 0.01 M Bis-Tris. $\mathrm{pH}=$ 7.4. Pulse increment: 0.02 V. Pulse period: $15 \mathrm{~s}$. Pulse width: 5 s. Sampling time: 100 ms. $T=298 \pm 3$ K.

Fig. 3

Cyclic voltamograms for the ion transport across a cholesterol-containing BLM in the presence of $500 \mathrm{nM}$ AmB. Start times of potential sweep: 0, 30, 120, 300 and $600 \mathrm{~s}$ (curves 1, 2, 3, 4, and 5) after the addition of AmB. Potential scanning rate: $50 \mathrm{mV} \mathrm{s}^{-1}$. Electrolyte in $\mathrm{W} 1: 0.03 \mathrm{M} \mathrm{KCl}$, 0.01 M HEPES and 0.01 M Bis-Tris. Electrolyte in W2: 0.01 M KCl, 0.01 M HEPES and 0.01 M Bis-Tris. $\mathrm{pH}=7.4 . T=298 \pm 3 \mathrm{~K}$. 
Fig. 4

Time dependence of the glucose concentration in (A) the cholesterol-containing liposome suspension and (B) the cholesterol-free liposome suspension when the DMSO solution of 1.0 mM AmB was added until the AmB concentration became $10 \mu \mathrm{M}$. A solid and a dashed arrows indicate the point when AmB was added and the point when Triton $\mathrm{X}-100$ was added. $\mathrm{pH}=7.4$. $T=298 \pm 1 \mathrm{~K}$. The insets indicate the change in the glucose concentration on the addition of Triton X-100 in the outside solutions of (A) the cholesterol-containing liposomes and (B) the cholesterol-free liposomes that had been left under $277 \mathrm{~K}$ for $13 \mathrm{~h}$ after the addition of $10 \mu \mathrm{M}$ of AmB. $\mathrm{pH}=7.4 . T=298 \pm 1 \mathrm{~K}$.

Fig. 5

Time dependence of absorbance at $408 \mathrm{~nm}$ (curve 1) and absorbance at $326 \mathrm{~nm}$ (curve 2) when 1.0 mM AmB in the DMSO was added to the NaPB solution (pH7.4). The final concentration of AmB: $10 \mu \mathrm{M}$. An arrow indicates the point at which AmB dissolved in DMSO was added.

The insets indicate absorbance spectra of (A) $10 \mu \mathrm{M}$ AmB in DMSO solution and (B) $10 \mu \mathrm{M}$ AmB solution in the NaPB solution (pH 7.4) containing 1.0 \% DMSO. 


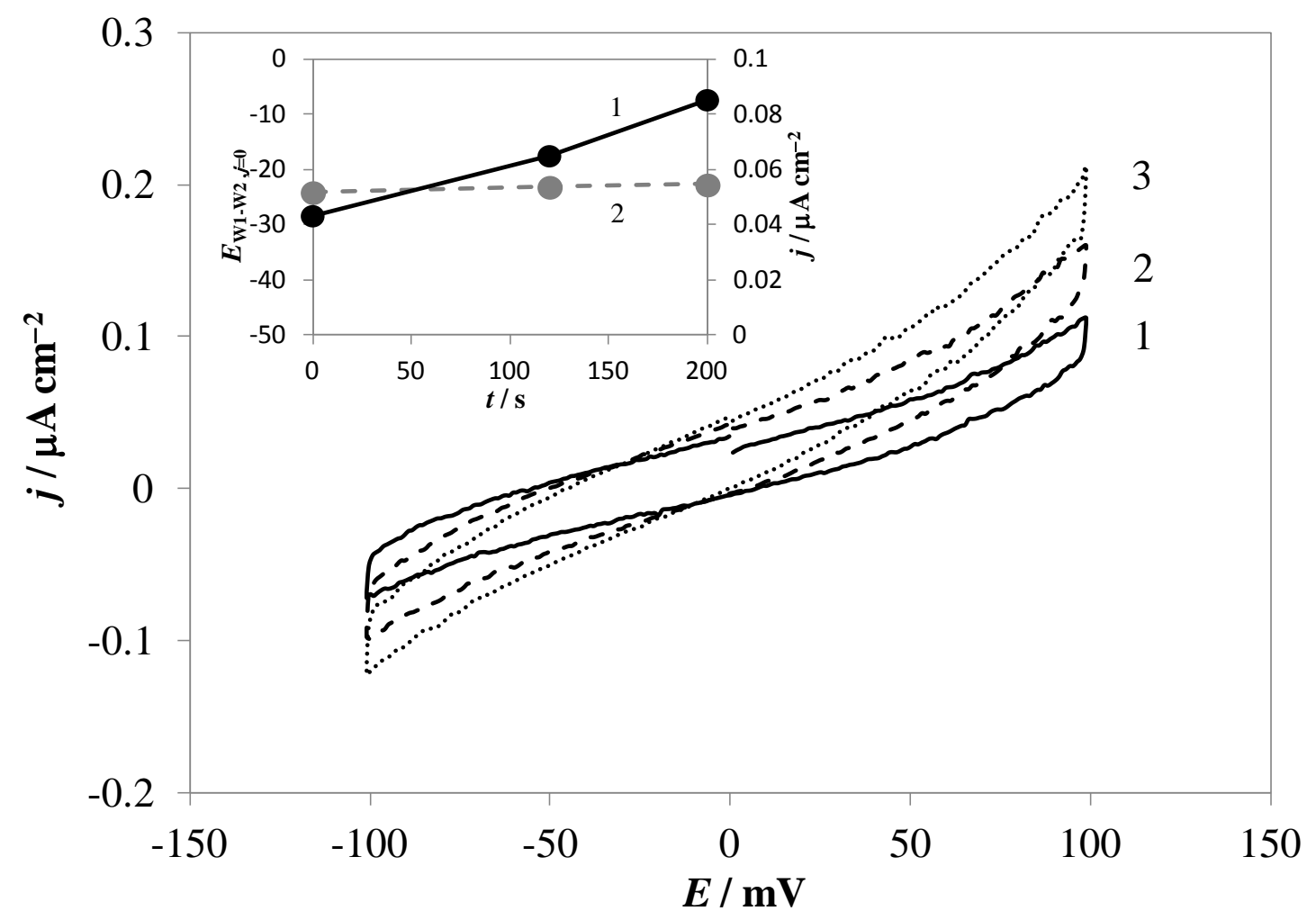

Fig. 1 H. Kagohashi, et al. 


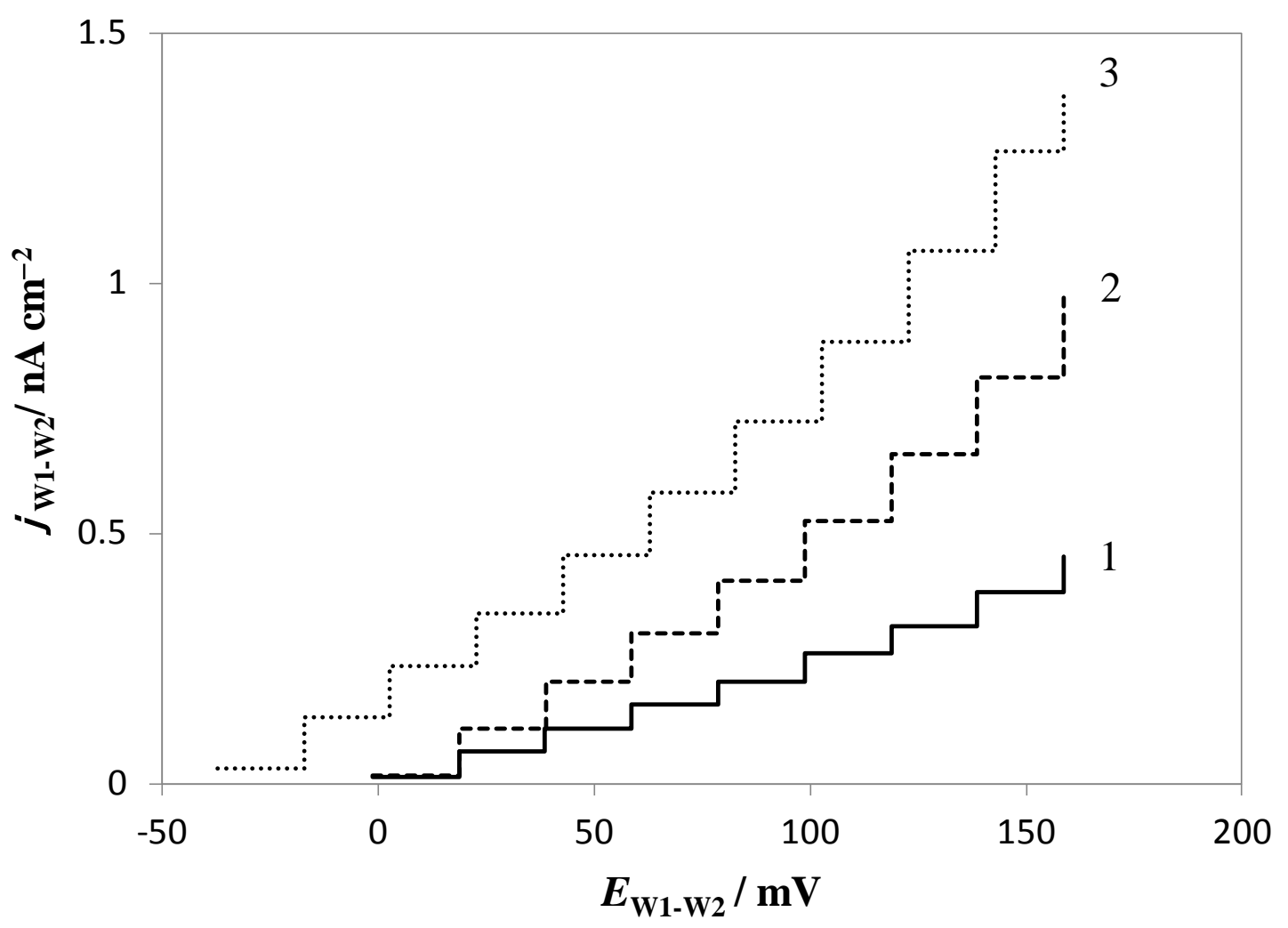

Fig. 2 H. Kagohashi, et al. 




Fig. 3 H. Kagohashi, et al. 


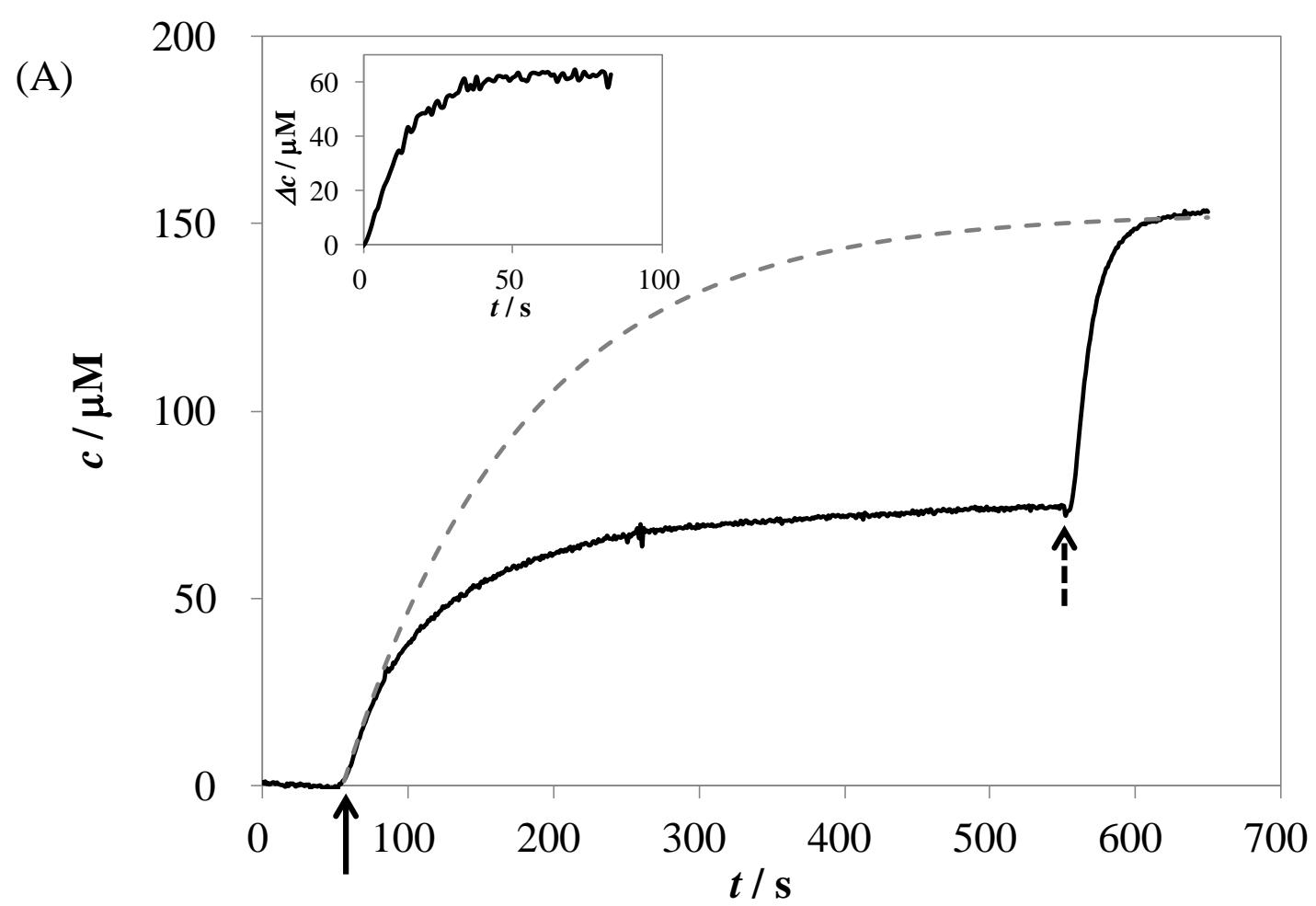

(B)

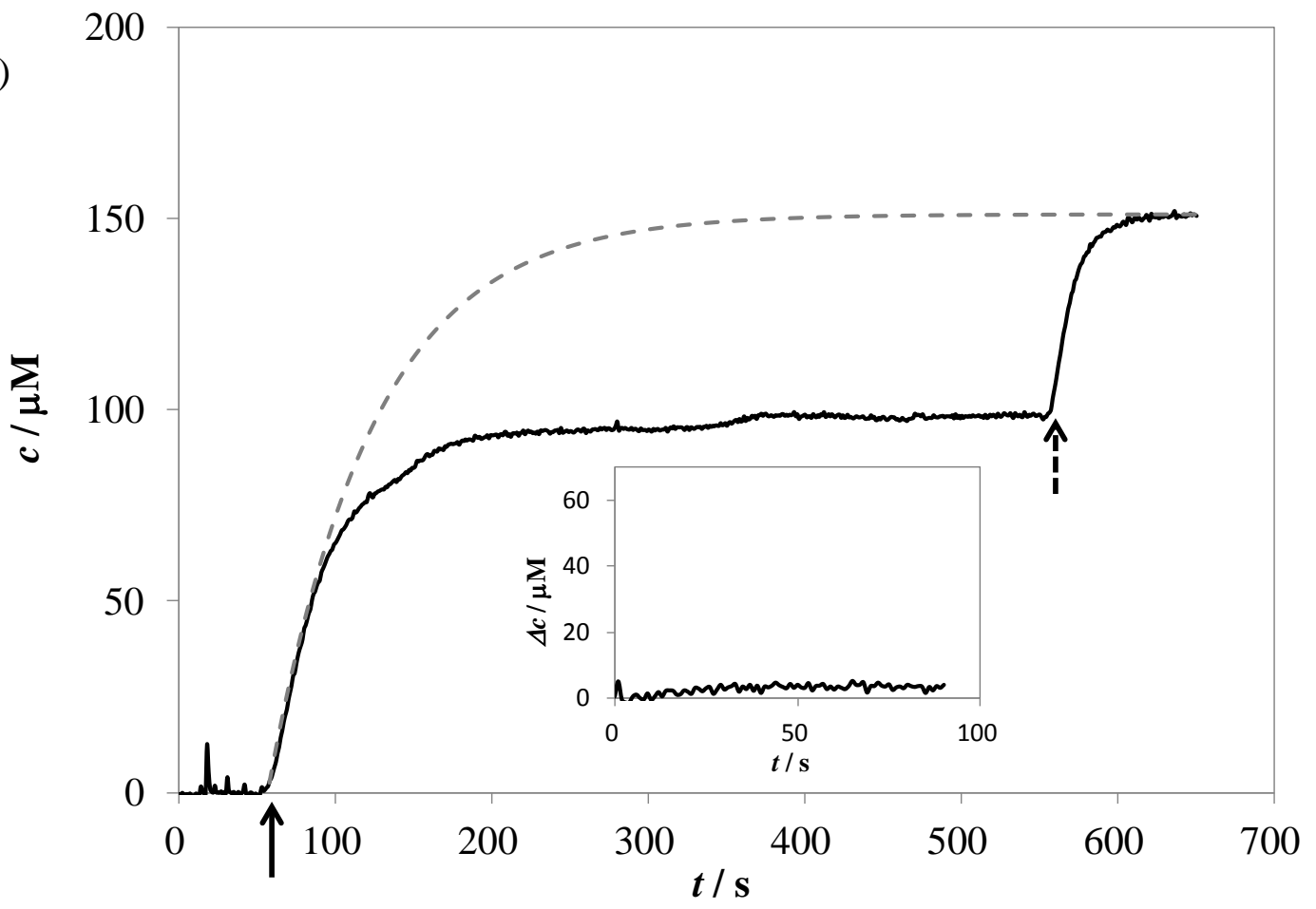

Fig. 4 H. Kagohashi, et al. 




Fig. 5 H. Kagohashi, et al. 
Facilitated Transport of Ions and Glucose by Amphotericin B across Lipid Bilayers in the Presence or Absence of Cholesterol

Hiroki Kagohashi, Osamu Shirai*, Shintaro Kubota, Yuki Kitazumi, and Kenji Kano

Division of Applied Life Sciences, Graduate School of Agriculture, Kyoto University, Sakyo, Kyoto 606-8502, Japan.

\section{Supporting Information}

\section{The fabrication methods of the sensors of glucose or NADH}

A glucose oxidase/peroxidase/ferrocene-immobilized electrode was fabricated in the following method [15]. The mixture of $500 \mathrm{mg}$ of graphite powder, $100 \mathrm{mg}$ of ferrocene (Fc) and $90 \mu \mathrm{L}$ of paraffin oil was tightly packed on the surface of the carbon paste electrode, which was purchased from Bioanalytical Systems (inner diameter $\phi=3.0 \mathrm{~mm}$ ). Then, $2.0 \mu \mathrm{L}$ of $3.0 \mathrm{mg}$ $\mathrm{mL}^{-1}$ glucose oxidase (from Aspergillus sp.) aqueous solution, $2.0 \mu \mathrm{L}$ of $3.0 \mathrm{mg} \mathrm{mL}^{-1}$ peroxidase (from Horseradish) aqueous solution, $1.0 \mu \mathrm{L}$ of $0.50 \mathrm{mg} \mathrm{mL}^{-1}$ bovine serum albumin aqueous solution and $1.0 \mu \mathrm{L}$ of $0.10 \%$ glutaraldehyde ethanol solution were put on the electrode surface. After the solvent was evaporated, the electrode surface was covered with a cellulose ester membrane (Spectrum Laboratories, Inc., molecular weight cut off 1000), and this membrane was fixed tightly with an O-ring and a nylon net. The electrode was stored at $4{ }^{\circ} \mathrm{C}$ in the $\mathrm{NaPB}$ solution (pH 7.4) when not in use. The reactions of the glucose-sensing electrode are:

$$
\begin{aligned}
& \beta \text {-D-Glucose }+\mathrm{O}_{2} \rightarrow \text { D-Glucono- } \delta \text {-lactone }+\mathrm{H}_{2} \mathrm{O}_{2} \quad \text { (glucose oxidase) } \\
& \mathrm{H}_{2} \mathrm{O}_{2}+2 \mathrm{Fc}+2 \mathrm{H}^{+} \rightarrow 2 \mathrm{H}_{2} \mathrm{O}+2 \mathrm{Fc}^{+} \quad \text { (peroxidase) } \\
& \mathrm{FC}^{+}+\mathrm{e}^{-} \rightarrow \mathrm{Fc}
\end{aligned}
$$


The fabrication method of the glucose dehydrogenase/1-methoxy PMS-immobilized electrode is noted below. The electrode reactions are written below.

$$
\begin{aligned}
& \beta-\mathrm{D}-\mathrm{Glucose}+\mathrm{NAD}^{+} \rightarrow \mathrm{D}-\text { Glucono- } \delta \text {-lactone }+\mathrm{NADH}+\mathrm{H}^{+} \text {(glucose dehydrogenase) } \\
& \mathrm{NADH}+\mathrm{PMS}^{+} \rightarrow \mathrm{NAD}^{+}+\mathrm{PMSH} \\
& \mathrm{PMSH} \rightarrow \mathrm{PMS}^{+}+2 \mathrm{e}^{-}+\mathrm{H}^{+}
\end{aligned}
$$

The mixture of $100 \mathrm{mg}$ of graphite powder, $20 \mathrm{mg}$ of 1-methoxy PMS and $18 \mu \mathrm{L}$ of paraffin oil was tightly packed on the surface of the carbon paste electrode. In addition, $2.0 \mu \mathrm{L}$ of $5.5 \mathrm{mg}$ $\mathrm{mL}^{-1} \mathrm{NAD}$-dependent glucose dehydrogenase (from Microorganism) aqueous solution, $1.0 \mu \mathrm{L}$ of $0.50 \mathrm{mg} \mathrm{mL}^{-1}$ bovine serum albumin aqueous solution and $1.0 \mu \mathrm{L}$ of $0.10 \%$ glutaraldehyde ethanol solution were dropped on the electrode surface. After the electrode was dried, the electrode surface was protected in a similar manner as the glucose oxidase/peroxidase/ferrocene-immobilized electrode.

\section{Durability of liposomes on the addition of AmB}

Figure S2 indicates time dependence of the current derived from $\mathrm{NAD}^{+}$in (A) the liposome suspension containing cholesterol and (B) the cholesterol-free liposome suspension when 1.0 $\mathrm{mM}$ AmB in DMSO was added until the AmB concentration became $10 \mu \mathrm{M}$. A solid arrow shows the point at which AmB was added. Dashed arrow denotes the point at which Triton $\mathrm{X}-100$ was added. Thus, $\mathrm{NAD}^{+}$cannot permeate across liposomal membranes containing AmB regardless of the presence and absence of cholesterol. This indicates that liposomes do not break on the addition of AmB. Therefore, we regard that the leakage of glucose in the presence of AmB was caused by the carrier-type and/or the channel-type transports. 


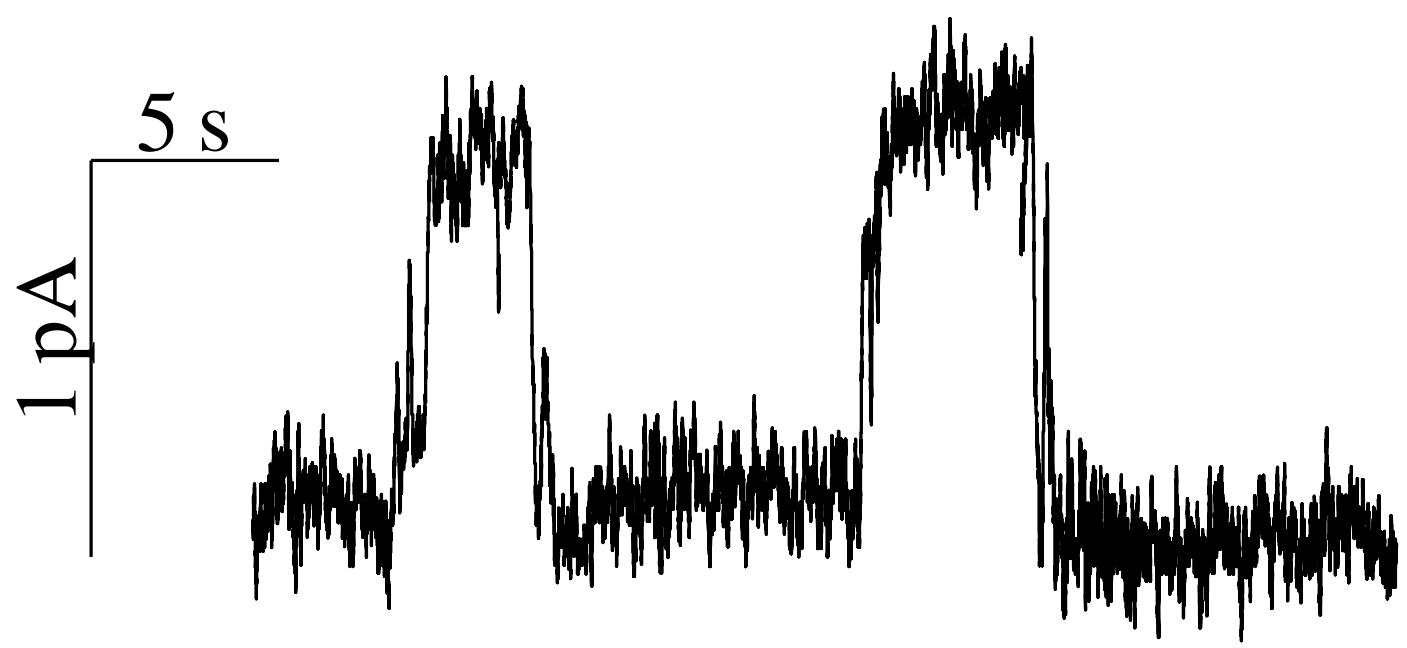

Fig. S1

The current fluctuation by the AmB channel in the BLM when W1 and W2 contained $0.3 \mathrm{M}$ $\mathrm{KCl}$ and $10^{-9} \mathrm{M}$ AmB. $T=298 \pm 1 \mathrm{~K}$. 

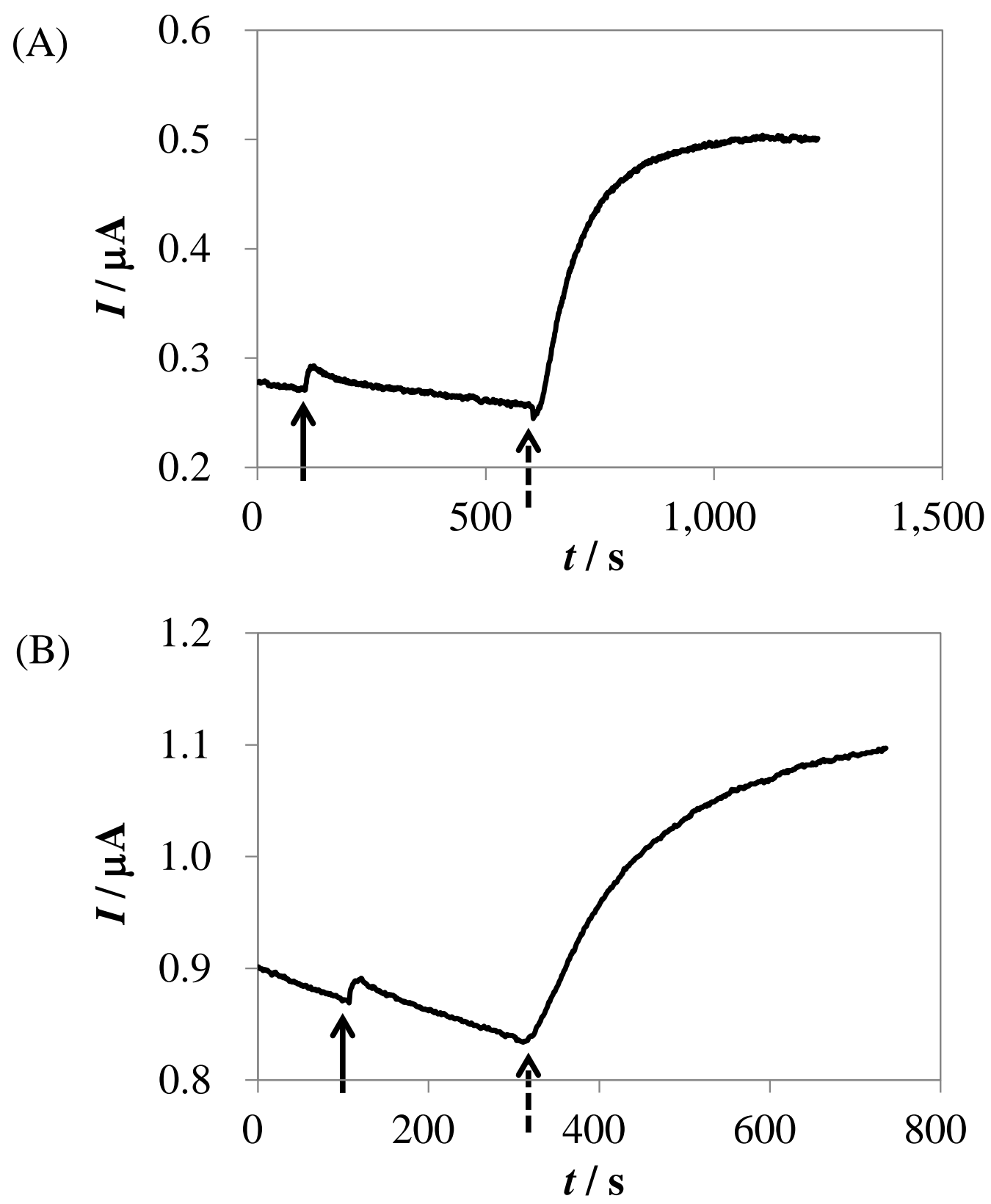

Fig. S2

Time dependence of the current derived from NADH which flowed out of (A) the cholesterol-containing liposome suspension and (B) the cholesterol-free liposome suspension, when $1.0 \mathrm{mM}$ AmB in DMSO was added (the final AmB concentration: $10 \mu \mathrm{M}$ ). Solid arrows indicate the point when AmB was added, while dashed arrows indicate the point when Triton X-100 was added. $\mathrm{pH}=7.4 . T=298 \pm 1 \mathrm{~K}$. 




Fig. S3

Time dependence of the glucose concentration in the cholesterol-free liposome suspension when $10 \mu \mathrm{L}$ of $1.0 \mathrm{mM}$ AmB in DMSO was added to $1 \mathrm{~mL}$ of liposome suspension (curve 1 ) and 10 $\mu \mathrm{L}$ of $1.0 \mathrm{mM}$ AmB dissolved in the NaPB solution containing $10 \%$ DMSO was added to it (curve 2). Solid arrows indicate the point at which AmB was added and dashed arrows indicate the point at which Triton X-100 was added. $c^{\infty}$ : the glucose concentration in the liposome suspension after Triton X-100 was added. $\mathrm{pH}=7.4 . T=298 \pm 1 \mathrm{~K}$. 